\title{
Power Allocation for Half-duplex Relay-based D2D Communication with QoS guarantee
}

\author{
Hui Dun, Fang Ye* and Shuhong Jiao \\ College of Information and Communication Engineering, Harbin Engineering University, Harbin, \\ Heilongjiang Province, 150001, China \\ [e-mail: m18846198960@163.com] \\ *Corresponding author: Fang Ye
}

Received November 29, 2017; revised March 24, 2018; accepted April 9, 2018;

published March 312019

\begin{abstract}
In the traditional cellular network communication, the cellular user and the base station exchange information through the uplink channel and downlink channel. Meanwhile, device-to-device (D2D) users access the cellular network by reusing the channel resources of the cellular users. However, when cellular user channel conditions are poor, not only D2D user cannot reuse its channel resources to access the network, but also cellular user's communication needs cannot be met. To solve this problem, we introduced a novelty D2D communication mechanism in the downlink, which D2D transmitter users as half-duplex (HD) relays to assist the downlink transmission of cellular users with reusing corresponding spectrum. The optimization goal of the system is to make the cellular users in the bad channel state meet the minimum transmission rate requirement and at the same time maximize the throughput of the D2D users. In addition, i for the purpose of improving the efficiency of relay transmission, we use two-antenna architecture of D2D relay to enable receive and transmit signals at the same time. Then we optimized power of base station and D2D relay separately with consideration of backhaul interference caused by two-antenna architectures. The simulation results show that the proposed HD relay strategyis superior to existing HD and full-duplex (FD) models in the aspects of system throughput and power efficiency.
\end{abstract}

Keywords: Device-to-Device, relay assist, Half-Duplex, two-antenna infrastructure 


\section{Introduction}

C onventional cellular networks are based on two-hop transmission mode from cellular users to base stations or core networks to cellular users. Such a network architecture facilitates stable network coverage and provides more efficient voice services [1]. However, with the advent of the 5G era, the types of services in mobile communication networks are also changing, and more data stream services and local services are exploding. This puts more demands on the mobile cellular network [2]. In order to meet the needs of the next generation of mobile communications, many new technologies have been proposed [3]. Among them, D2D communication allows users to communicate directly without the need to forward data via the base station, which not only can improve the data transmission rate, but also increase the system spectrum efficiency, which has attracted extensive attention from the academic community and industry $[4,5]$. However, the introduction of D2D communication brings a more complex interference environment to mobile cellular networks. In order to solve this problem, more advanced algorithms involved with power allocation, interference management and resource allocation are needed [6].

A great deal of existing study theory focus on the scenario of D2D reuse mode in which the D2D user acts as a secondary user and accesses the cellular network by reusing the spectrum resources of the cellular user. Various strategies have been proposed to manage the mutual interference between cellular and D2D users in the resource reuse scenario [7]. In [8] a resource allocation algorithm based on location information was proposed. By delineating the limited area for each cellular user, D2D users falling within this area cannot reuse their spectrum resources, thus protecting D2D. users are subject to interference from cellular users and also reduce algorithm complexity. However, the cellular user can act as a candidate reusing pair for D2D user only if the cellular user's transmission requirement is guaranteed. When the primary user, namely, the cellular user suffer from severe channel fading or at edge of the cell, the transmission demand of the cellular user itself cannot be satisfied. Its spectrum resources cannot be reused. This not only reduces the transmission quality of cellular users, but also reduces the access rate of D2D users. In [9], a resource allocation mechanism based on the Stackelberg game is proposed. With the consideration of the access number of D2D appliance, D2D users are allowed to reusing multiple cellular users' spectrum resource at the same time. Then D2D users purchase the interference quota from base station as a follower. But the algorithm only considers the case of uniform price, that is, each cellular user is assumed to be same sensitivity to interference. The simplified scenario of including single cellular user and single pair of D2D users is considered in [10], with maximizing the sum rate of cellular users and D2D users while guaranteeing the minimum transmission rate requirements of primary users. For more practical scenarios in which multiple cellular and multiple D2D users exist in single cellular network, the corresponding spectrum and power allocation algorithm are proposed in [11] and [12]. In [13], the author proposes a mobile cloud computing-based D2D access mechanism for heterogeneous cellular network scenarios, which improves the overall throughput of cellular networks. It should be noted that the aforementioned D2D strategies as underlaying mode, will create considerable interference to the cellular network, thereby affecting the quality of service (QoS) and quality of experience (QoE) requirements of cellular users whose transmission requirement should be satisfied in priority $[14,15]$. Especially for cellular users in deep fading or at the edge of the cell, it is not recommended to share their spectrum resources with D2D users.

In view of the above, a new D2D communication mechanism is proposed in which a D2D transmitter is used as a relay to assist cellular users in a bad channel state to transmit data while 
reusing their spectrum resources for D2D users' own data transmission. Such a transmission mechanism still makes sense in future small cell scenarios. Based on the relay-assisted D2D communication mechanism, some research results have been obtained. Through the browsing of the existing literature, we find that according to the different working modes of the relay, it can be divided into two categories, namely D2D relay assistance based on HD and D2D relay assist based on FD. In fact, some research results have been achieved for the D2D-assisted communication mode based on HD. In the HD mode, one transmission subframe is divided into two equal transmission slots, and the D2D relay completes the reception and forwarding of the cellular signals in the slots, respectively $[16,17]$. Then, in forwarding phase, D2D relay superimposes the received primary user and D2D communication signals, and then transmits this linear combined signal. In the superimposed signal, the weighting factor of primary user is transmission power allocated to cellular signal by the D2D relay. The remaining D2D relay transmit power is used to transmit D2D user signals. Since the direct transmission period from the base station to the cellular user has only one transmission time slot, the throughput of the primary user is cut down by $50 \%$. To make up for the throughput loss of primary user, the D2D relay splits total transmission power [18]. In the following transmission phase, primary user signal is forwarded. However, the authors in [19], the authors explicitly point out that base stations and D2D relays use maximum transmit power for signal transmission, which results in low energy efficiency of the system. Especially for D2D relay, its battery capacity is small, and its energy efficiency should be considered.

The disadvantage of HD mode is that spectral efficiency and energy efficiency are low [20]. Therefore, as a substitute for the HD mode, the FD relay forwarding method can improve both spectral efficiency and energy efficiency by being able to simultaneously transmit and receive signals [21]. In order to achieve simultaneous transmission and reception of transmission relays, D2D relays are usually equipped with a two-antenna architecture. However, since FD can achieve simultaneous transmission and reception, the D2D relay is easily affected by the backhaul link interference, which is generated by the relay transmit antenna and received by the relay receive antenna [22]. Although many signal processing methods have been proposed to eliminate backhaul link interference, they are still not negligible relative to Gaussian white noise [23]. In addition, it should be clearly pointed out that since the full duplex mode allows the D2D relay to transmit the primary user and D2D user signals on the same spectrum bandwidth, the two signals are mutually interfering signals. The work in [24] proposes a new FD mode is proposed, assuming that the sum of the transmission powers of the system is a constant. This assumption allows D2D relays to transmit signals in excess of their maximum transmit power, as long as the system consume constant overall power. Through such an equation assumption, the author obtains the exact expression of optimal transmit power in closed-form. Hence, the system performance is significantly improved. However, a more realistic assumption is that base stations and D2D relay users have separate transmit power constraints. Moreover, for the enhancement of the spectrum efficiency of HD mode, author in $[25,26]$ proposes the concept of virtual full-duplex, through two HD relays, to achieve simultaneous transmission and reception performance, and model the transmission process as a Markov chain.

Combining the advantages of HD and FD, we propose a novelty HD mode of two-antenna architecture. Considering the architecture of the two antennas, the D2D relay can still transmit and receive signals simultaneously in the HD mode, which improves the transmission rate of D2D communication and improves the spectrum utilization of the system in comparison to the traditional HD mode. For the mitigation of the backhaul interference which is generated due to transmitting and receiving signals simultaneously of D2D relay, we have allocated power to 
the base station and the D2D relay, and obtained a closed expression of the optimal transmission power. By comparing with existing work, this article has made some contributions in the following aspects

(1) Considering the more efficient transmission brought by the two-antenna architecture, we propose a new HD D2D relay assist mode. We assume that the D2D transmitter has a two-antenna infrastructure, namely, a receiving antenna and a transmitting antenna. Therefore, the D2D relay has the function of simultaneous transmission and reception. At the same time, the backhaul link interference caused by this is considered.

(2) Forthermore, we consider each node to have independent maximum transmit power constraints. We deduce optimal transmit power of each node, and give a closed expression. Specifically, we convert the D2D relayed forwarding signal from a mixed signal of a primary user and a D2D user signals to a separate cellular user signal. As we explained earlier, in the mixed signal transmitted by D2D relay, both signals will deteriorate the counterpart SINR, which will reduce transmission rate of bothlinks. Therefore, separate transmission of primary user and D2D user signals result in more efficient spectral efficiency.

(3) Unlike some of the research results we have already done on relay assistance, in this article. We mainly study a new type of HD relay, while in [23], we give the optimal power selection in FD mode. Moreover, in [27], we introduce a slot splitting factor for the existing HD mode, assuming that one transmission frame can be arbitrarily split into two transmission slots of different sizes, but there are still limitations in some technical conditions.

The structure of this articleis arranged as follows. In Section II, we give the system model and explain proposed HD relay-based D2D aidedmechanism. in section III, we use the power allocation strategy, give the optimal transmit power of each node in the system, and give its closed expressions. Performance simulation is given for our proposed HD mechanism in section IV. Finally, we give some useful conclusions in section V.

\section{System Model}

This article studiesa single-cell downlink scenario in whichthe macro base station is located in the center of network, single primaryuser and singleD2D users. It is assumed that the cellular user is located at the edge of the cell or is in a deep fading state. Therefore, the traditional straight-through link from base station to the primaryuser cannot meet transmission rate requirement of cellular user. The D2D user accesses cellular network by reusing subchannelof primaryuser. Because the channel quality of cellular user itself is not good, the traditional D2D user can directly reuse the cellular user resources. Therefore, D2D users need to assist the transmission of cellular users when reusing cellular user resources. The system model is illustrated in Fig. 1. In this model, B represents the macro base station, $\mathrm{C}$ represents the cellular user located in the edge of network, R represents a D2D transmitting user, and serves as the secondary relay for primaryuser, and D represents D2D receiving user respectively. Different from the existing model, it is assumedthat relay-baseduser, that is, the D2D transmitting user is equipped with an independent transmitting and receiving antenna and cache the data which is transmitted to D2Dreceiver. In addition, our proposed strategyuses a half-duplex mode to split one transmission frame into two transmission phases in the time domain. In the first transmission phase, , the macro base station broadcasts a cellular user signal over a channel that is pre-assigned to the cellular user. Obviously, due to the 
underlaying the spectrum resource of primaryuser, D2D relay receives this signal at the same time. Moreover, also in the first transmission phase, it is assumed that D2D relay, the D2D transmitting user, transmits its own signal to the D2D receiving user. Obviously, D2D relays will inevitably be interfered with by the backhaul link due to simultaneous signal transmission and reception. In followingtransmissiontime slot, D2D transmitter user decodes and forwards this signals to primaryuser. It should be noted that in this scenario, the channel resources of the cellular user have been allocated by the base station.

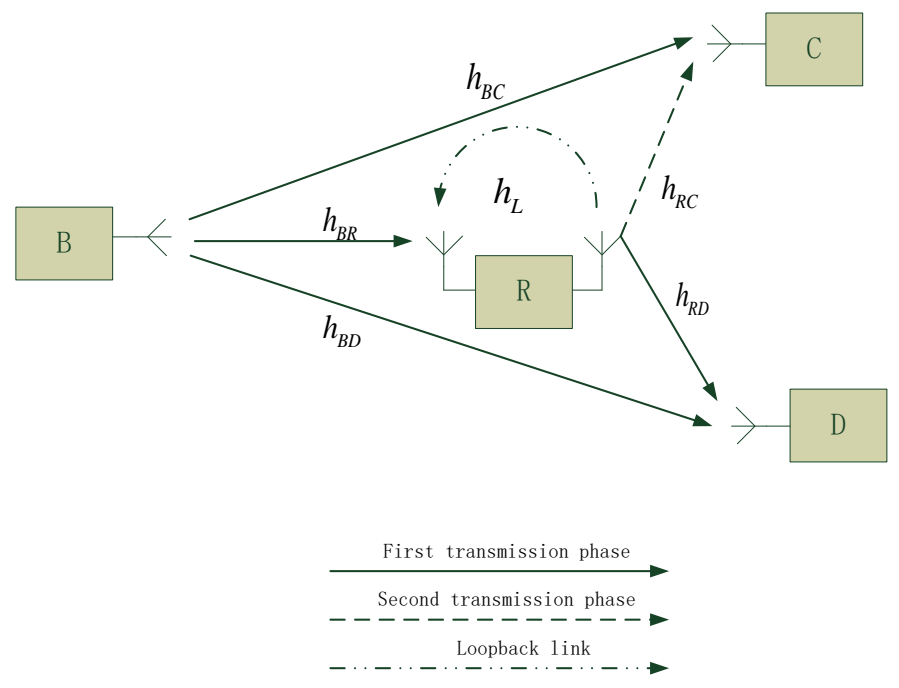

Fig. 1. Relay-aided D2D communication model in HD mode.

We consider the downlink frequency division duplex system. System bandwidth is $W \mathrm{~Hz}$. We use $h_{B C}, h_{B R}, h_{B D}, h_{R C}, h_{R D}$ and $h_{L}$ to describes the channel state information from macro base station to primary user, macro base station to D2D transmitter user, macro base station to D2D receiver user, D2D transmitter user to primary user, D2D transmitter user to corresponding receiver user and backhaul interference channel of D2D transmitter user in term of channel impulse response respectively. Therefore, the corresponding channel gain-to-noise ratios can be describe as follow

$$
\begin{gathered}
\gamma_{B R}=\left|h_{B R}\right|^{2} / \sigma^{2}, \gamma_{B C}=\left|h_{B C}\right|^{2} / \sigma^{2}, \gamma_{B D}=\left|h_{B D}\right|^{2} / \sigma^{2} \\
\gamma_{R C}=\left|h_{R C}\right|^{2} / \sigma^{2}, \gamma_{R D}=\left|h_{R D}\right|^{2} / \sigma^{2}, \gamma_{L}=\left|h_{L}\right|^{2} / \sigma^{2}
\end{gathered}
$$

Where $\sigma^{2}$ describes white Gaussian noise. Moreover, we use to $p_{B}$ and $p_{R}$ to describe transmitting power of macro base station and D2D transmitter userrespectively. It should be pointed out that we use normalized $p_{B}$ and $p_{R}$ for simplification which also satisfy constraints of $0 \leq p_{B} \leq 1$ and $0 \leq p_{R} \leq 1$ indenpently rather than constant aggregate transmitting power condition a as done in [23].

It should be noticed that all of system parameters aforementioned can be described as the signal-to-noise ratio (SNR) of corresponding channels with using unit transmit power. We assume that all channels involved in system are quasi-static flat fading, which remains constant for at least one frame. Every node has perfect channel state information. 


\section{HD relaying based D2D communication scheme}

In traditional cellular network, cellular user wants to transmit data only through direct communication with the base station, while such a transmission rate is low. Unfortunately, such a situation is not a small probability event. When a cellular subscriber is at the edge of a cell or is in a deep fading state, the quality of service of cellular users is often not guaranteed.

In a traditional cellular network scenario, the transmission rate of cellular user can be described as

$$
R_{B C}^{D T}=W \log _{2}\left(1+\frac{p_{B} h_{B C}}{\sigma^{2}}\right)
$$

Assuming that the right side of this equation divides by $\sigma^{2}$ as done in (1), we can reshape (2) as $R_{B C}^{D T}=W \log _{2}\left(1+\gamma_{B C}\right)$.

We consider the constraints of meeting the minimum transmission rate requirements $R^{r e}$ of cellular users. However, it is pretty common that cellular user locates in cell edge or suffered from deep fading or shadowing. In this scenario, the quality of service of the cellular user cannot be satisfied even if the base station uses the maximum transmit power for signal transmission , namely,

$$
R^{r e}>W \log _{2}\left(1+\gamma_{B C}\right)
$$

It means that straightforward communication from macro base station to primary user cannot meet the quality of service (QoS) of cellular user in term of transmission rate. Therefore, we look forward to proposed a novelty D2D relaying-assist strategy with guaranteeing rate requirement of primary user.

Different from the existed HD relay mode, we let the D2D relay send D2D signal during the first time slot rather than transmit the combination signals of relaying signal and D2D signal during the second time slot which degrades power efficiency of D2D relay with using a power splitting factor as in [23]. With equipped with two-antenna infrastructure, the HD relaying based D2D protocol we proposed allow the D2D relay transmit and receive signals simultaneously in the first transmission phase.

We denote $s_{C}$ and $s_{D}$ as the signals intended to send to primary user and D2D receiver. We have $E\left\{s_{C}\right\}=1$ and $E\left\{s_{D}\right\}=1$ where $E\{\cdot\}$ describes the expectation function. Furthermore, we can describe the expression of receiving signals at D2D transmitter and D2D receiver as follow with consideration of backhaul interference channel from D2D relay transmitting antenna to the receiving antenna

$$
\begin{aligned}
& r_{\mathrm{R}}=\sqrt{p_{B}} h_{B R} s_{C}+\sqrt{p_{R 1}} h_{L} s_{D}+n_{0} \\
& r_{D}=\sqrt{p_{B}} h_{B D} s_{C}+\sqrt{p_{R 1}} h_{R D} s_{D}+n_{0}
\end{aligned}
$$

where $n_{0}$ describes the interference signal and $p_{R 1}$ denotes the transmitting power of D2D transmitter in the first time slot.

Therefore, we can describe the signal-to-interference and noise ratios (SINR) of D2D transmitter user and D2D receiver user during the first transmission time slot as

$$
\gamma_{R}=\frac{p_{B}\left|h_{B R}\right|^{2}}{p_{R 1}\left|h_{L}\right|^{2}+\sigma^{2}}
$$




$$
\gamma_{D}=\frac{p_{R 1}\left|h_{R D}\right|^{2}}{p_{B}\left|h_{B D}\right|^{2}+\sigma^{2}}
$$

We let the numerator and denominator on the right sides of those equations divided by $\sigma^{2}$. Therefore, the SINR of D2D relay and D2D receiver user described above can be transformed as

$$
\begin{aligned}
& \gamma_{R}=\frac{p_{B} \gamma_{B R}}{p_{R 1} \gamma_{L}+1} \\
& \gamma_{D}=\frac{p_{R 1} \gamma_{R D}}{p_{B} \gamma_{B D}+1}
\end{aligned}
$$

During the following transmission time slot, D2D transmitter user decodes signal of primary user received during the first time slot and sends to corresponding receiving user by using transmit power of $p_{R 2}$. Then these receiving signals at primary user can be described as

$$
r_{C}=\sqrt{p_{R 2}} h_{R C} s_{C}+n_{0}
$$

Just like we did previously, the SINR of primary user during the following transmission time slot can be described as

$$
\gamma_{C}=\frac{p_{R 2}\left|h_{R C}\right|^{2}}{\sigma^{2}}
$$

It is obvious that the channel capacity of link from D2D transmitter to primary user has tight correlation with transmit power fo relay and corresponding channel gain. Therefore, it should be pointed out that the D2D relay-based strategy in this article makes a difference from the existing related works, which are all forwarding mixed signals of primary user and D2D user. Nevertheless, the D2D transmitter user forwards mixed signals will result in inferior SINR for each user. We simplify the expression of equation (11) by dividing $\sigma^{2}$ for numerator and denominator. Than the expression of equation (11) can be described as

$$
\gamma_{C}=p_{R 2} \gamma_{R C}
$$

It should be noted that the instantaneous end-to-end SINR of signals transmitting from base station to primary user in proposed HD strategy is determined by the worse hop, which can be described as

$$
\gamma_{H D}=\min \left\{\gamma_{R}, \gamma_{C}\right\}
$$

In this article, we assumed that macro base station has the perfect knowledge of channel state information for every involved link. The Shannon's formula give a upper bound of achievable transmission rate. Therefore, the achievable transmission rates of primary user can be calculated respectively as

$$
\begin{aligned}
& R_{C}^{H D}=\frac{1}{2} W \log _{2}\left(1+\gamma_{H D}\right) \\
& R_{D}^{H D}=\frac{1}{2} W \log _{2}\left(1+\gamma_{D}\right)
\end{aligned}
$$

Finally, for the sake of satisfying rate requirement of primary userin proposed D2D relay strategy, the achievable rate of cellular user should no less than requirement rate with proposed D2D relay strategy which can be described as 


$$
\frac{1}{2} W \log _{2}\left(1+\gamma_{H D}\right) \geq R^{r e}
$$

Therefore, we can deduce the value scope of $\gamma_{H D}$ as

$$
\gamma_{H D} \geq 2^{2 R^{r e} / W}-1
$$

Inour study, we want to make the largest improvement in term of throughput for the D2D communication link with the QoS guarantee for cellular user in term of minimum rate requirement. Therefore, the objective function can be described as follow

$$
\begin{gathered}
\left(p_{B}^{*}, p_{R 1}^{*}, p_{R 2}^{*}\right)=\arg \max _{\left(p_{B}, p_{1}, p_{R 2}\right)} \frac{1}{2} W \log _{2}\left(1+\gamma_{D}\right) \\
\text { Subject to } R_{C}^{H D}=R^{r e} \\
p_{B} \leq 1 \\
p_{R 1} \leq 1 \\
p_{R 2} \leq 1
\end{gathered}
$$

Where $\left(p_{B}{ }^{*}, p_{R 1}{ }^{*}, p_{R 2}{ }^{*}\right)$ describes optimal power values for macro base station and D2D transmitter with satisfying all constraints in the first phase and in the second phase.

In the following step, we solve the aboved optimization problem to maximize achievable rate of D2D communication. For coverage extension ofnetwork, base station should always adopt maximal power as the optimal transmit power, which is same to the traditional HD mode scheme, i.e. $p_{B}=1$. It is obviously that the first relay channel will have the best SINR, namely, $\gamma_{R}$. In the following, we will focus on power optimization for D2D transmitter alone.

Due to the fact that the bottleneck constraint for instantaneous end-to-end SINR for a relayed signal, which uses decode and forward mode by two hops is the hop with worse channel gain. Therefore, we will classify the power allocation problem by two different case as follow

Case one: The limited factor for achievable capacity of cellular user is first transmission hop because of severe loopback interference at D2D relay. As a result, we should make sure that $\gamma_{R}=\gamma_{H D}$ in the first place. By solving this equation, we can get

$$
p_{R 1}=\min \left\{1, \frac{\gamma_{B R}-\gamma_{H D}}{\gamma_{H D} \gamma_{L}}\right\}
$$

Moreover, in our proposed D2D relay mode, the forwarding cellular user signal is transmitted by the D2D relay alone rather than a linear combined signal. The throughput of D2D commnication link can not be improved with more transmit power of D2D relay. To the opposite, with a consideration to improve the power efficiency for the system, we set $\gamma_{C}=\gamma_{H D}$ as done before. Then we have

$$
p_{R 2}=\min \left\{1, \frac{\gamma_{H D}}{\gamma_{R C}}\right\}
$$

Case two: The limited factor for achievable capacity of cellular user is second transmission hop because of bad channel state of link between D2D transmitter and cellular user. Then we have $\gamma_{C}=\gamma_{H D}$ firstly. As done in case one, we have 


$$
p_{R 2}=\min \left\{1, \frac{\gamma_{H D}}{\gamma_{R C}}\right\}
$$

Moreover, to achieve largest transmitting capacity of D2D communication, we use $p_{R 1}=1$ as the transmission power of D2D transmitter for the first time slot.

\section{Simulation results}

In the part of simulation results, with using MATLAB simulation, it can be verified that this proposed HD mechanism improves the throughput and energy efficiency of the system while ensuring the quality of cellular users. The existing strategies as the traditional HD mode and FD mode done in [24] are chosen as the comparison algorithm. It can be demonstrated through simulations that the novelty D2D relay-aided communication strategy could achieve lager channel capacity when make a comparison with traditional HD mode and FD mode. Moreover, the proposed relay-based D2D communication ensure to satisfy rate requirement of cellular user at first. . The some partial important parameters of system such as rate requirement of primary user is equal to $R^{r e}=50 \mathrm{~kb} / \mathrm{s}$, and the bandwidth is $W=0.1 \mathrm{MHz}$. Moreover, we assume that $\gamma_{B R}=\gamma_{R C}=10 \mathrm{~dB}, \gamma_{R D}=30 \mathrm{~dB}$ and $\gamma_{B C}=\gamma_{B D}=-5 \mathrm{~dB}$, in which the bad channel state describes the channel suffered from deep fading or shadowing.

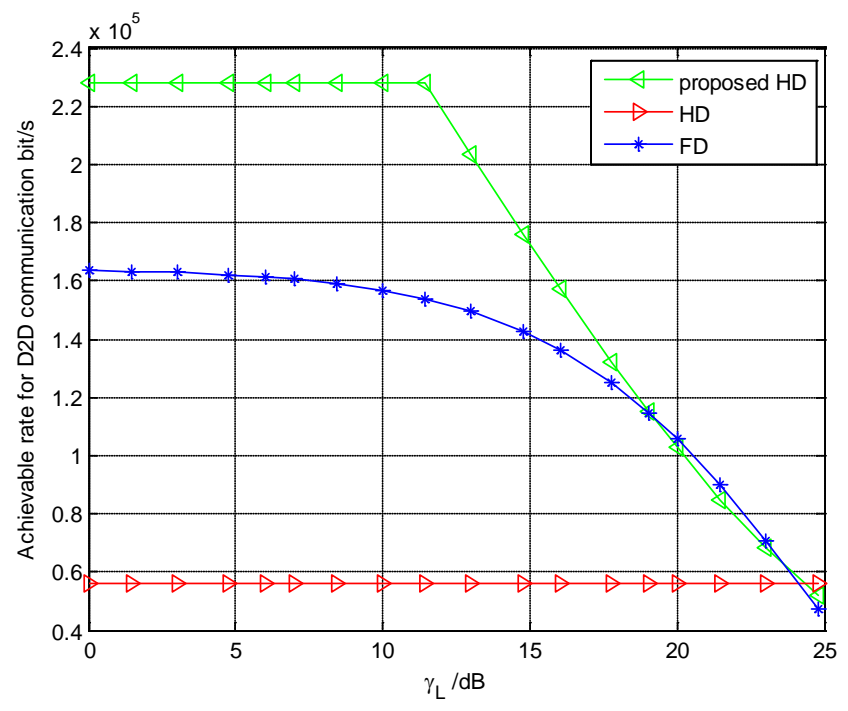

Fig. 2. Rate gain achievable by D2D communication with different loopback channel gain

In Fig. 2, the simulation resoult describes that how backhaul interference influence optimal transmit power at D2D transmitter. To get the precise curve, we set the coordinate scope of $\gamma_{L}$ ranging from $0 \mathrm{~dB}$ to $25 \mathrm{~dB}$. The simulation picture demonstrated that the achievable rate of D2D maintains constant and provides remarkable transmission rate achievement improvement with contrasting to traditional HD and FD scheme at $\gamma_{L} \leq 12 \mathrm{~dB}$. The reason is that the loopback interference isn't excessive and the D2D relay could transmit signals with usage of maximum power. When $\gamma_{L} \geq 12 \mathrm{~dB}$, the performance of proposed HD scheme decreases linearly, however, it's also provides significant performance improvement with comparison of traditional HD and equivalent to FD mode. 


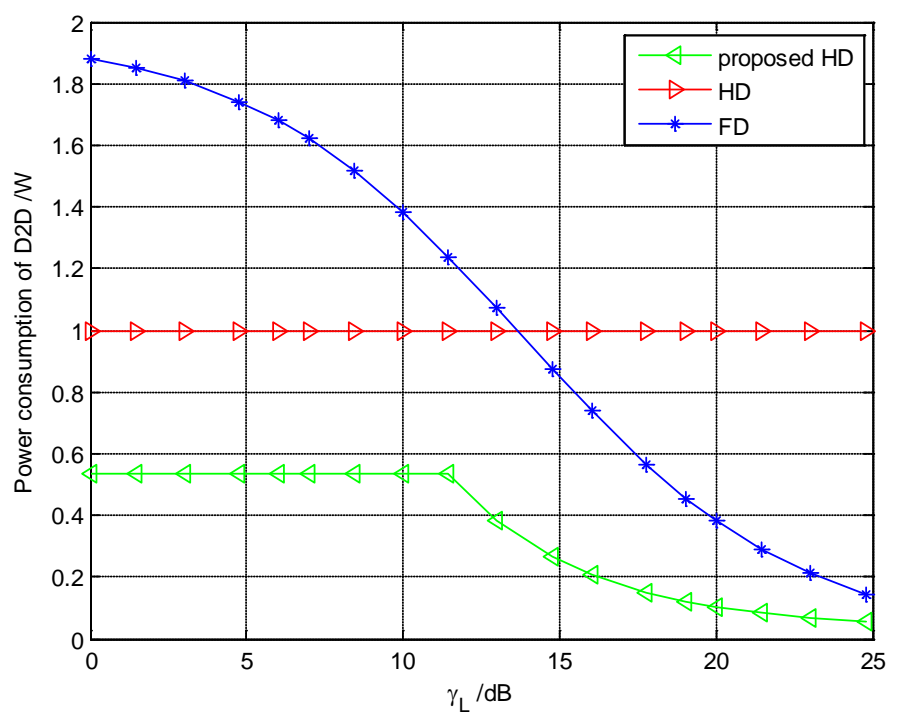

Fig. 3. Power consumption of D2D relay with different loopback channel gain

In Fig. 3, the simulation result describes the power consumption between those three relay modes. As we can see that in traditional HD mode, the D2D relay uses the maximum power all the time. Moreover, the FD scheme in [24] assume that the D2D relay could use transmission power great than 1 in which the total power of base station and D2D transmitter user is constant to 2. However, the proposed scheme allows D2D relay transmit the cellular user signals and its own signals in different time slots, which avoids transmitting the combinatorial signals with using a power splitting, factor. Because of this reason, the proposed scheme consumes fewer energy when guaranteeing rate requirement forprimary user, which ensures D2D transmitter enjoy a more attractive battery life.

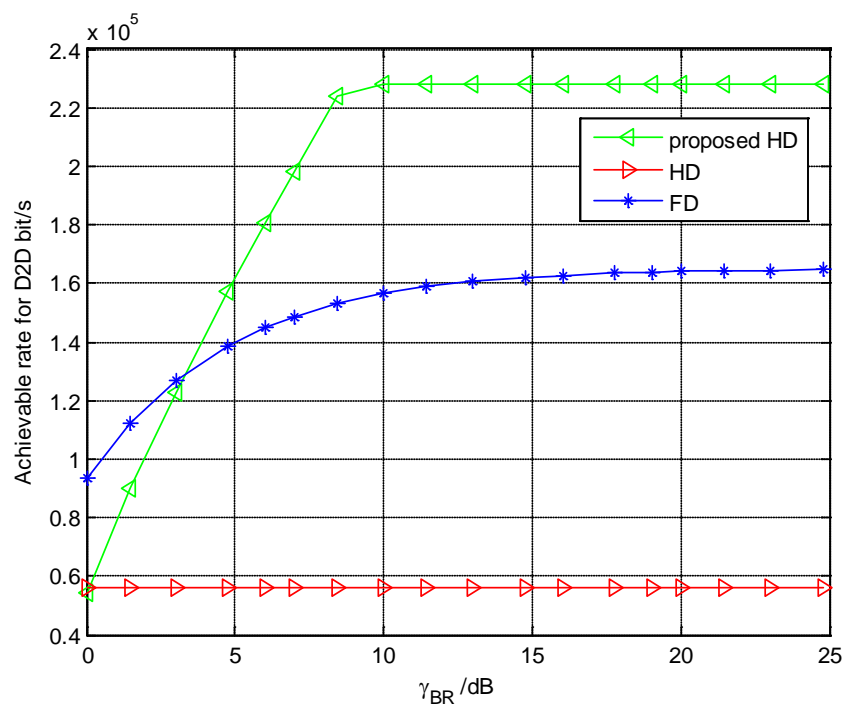

Fig. 4. Rate gain achievable by D2D communication with different channel gain from BS to D2D relay 
In Fig. 4, we show the impact of the variation of link between base station and D2D relay on performance of channel capacity for D2Duser. The channel gain of loopback link is fixed to $\gamma_{L}=10 \mathrm{~dB}$ and $\gamma_{B R}$ is vary from $0 \mathrm{~dB}$ to $25 \mathrm{~dB}$. The result shows that the proposed HD scheme enjoys a rapid growth tendency then both contrast algorithms and achieves significant rate gain as the enlargement of $\gamma_{B R}$ when $\gamma_{B R} \geq 3 \mathrm{~dB}$. The reason account for this is D2D relay can use the maximum power as channel quality improvement in the first hop.

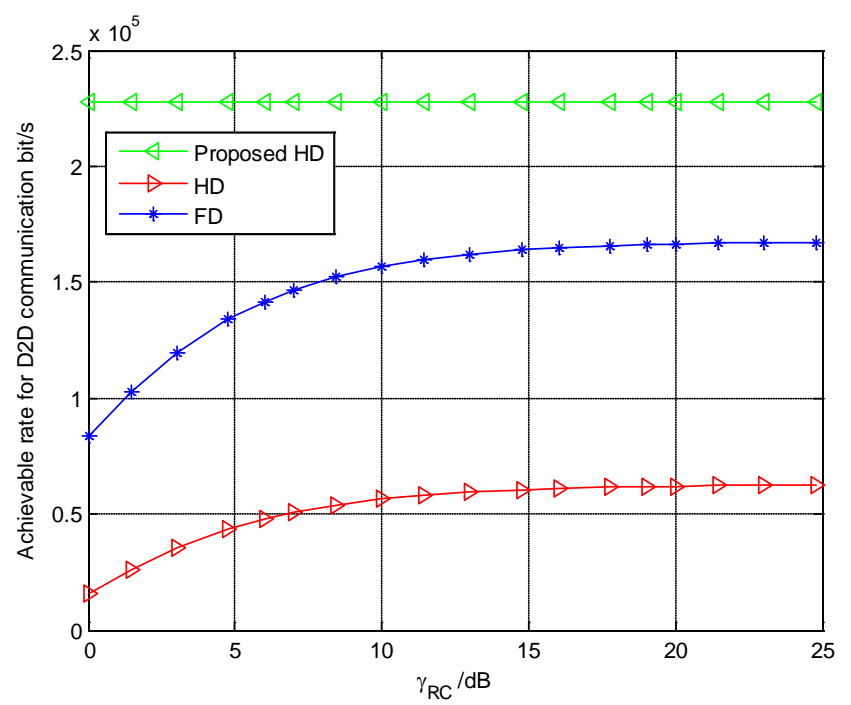

Fig. 5. Rate gain achievable by D2D communication with different channel gain from D2D relay to cellular user

Different from Fig. 4, Fig. 5 reveals the influence of channel gain between the D2D relay and cellular. As $\gamma_{R C}$ varies from $0 \mathrm{~dB}$ to $25 \mathrm{~dB}$, we can observe that the proposed scheme achieves biggest transmission rate improvement among the three algorithms and maintains as a constant. As the analysis we have done above, the D2D relay need allocate more power in the second phase to keep the achievable rate guaranteed when the channel gain of second hop is weak. However, it has no effect on the transmission power of the D2D relay in the first phase. In our proposed scheme, the D2D relay will transmit its own signals in the first phase with usage of $p_{R 1}$. Therefore, the advantage of our proposed algorithm is obviously as the performance of system can be maintained when $\gamma_{R C}$ is deterioration.

\section{Conclusions}

In the article, a HD relay-aided D2D strategy is proposed with underlying cellular network' downlink spectrum. Different from existing HD relay mode, it is assumed that D2D transitter user has a two-antenna infrastructure which allows the D2D relay communicates bi-directionally with cellular user and D2D receiver. Moreover, we divided one frame into two transmission phases and the D2D relay forwards the cellular user and its own signals in different phases. Finally, optimal transmission power of D2D transmitter user is resolved with precisely expression and simulation results show the verification that proposed relay-aided D2D communication strategy makes great improvement on channel capacity of D2D and use 
less transmit power in comparison to the benchmark which enhances energy efficiency for overall system and extends lifetime of battery.

\section{Acknowledgments}

The paper is funded by the International Exchange Program of Harbin Engineering University for Innovation-oriented Talents Cultivation. And this work is partially supported by the National key research and development program of China (Grant No.2016YFF0102806), the National Natural Science Foundation of China (Grant No.61701134), the Natural Science Foundation of Heilongjiang Province, China (Grant No. F2017004)

\section{References}

[1] X. Lin, J. Andrwes, A. Ghosh and R. Ratasuk, "An overview of 3GPP device-to-device proximity services,” IEEE Communications Magazine, vol. 52, no. 4, pp. 40-48, April, 2014.

Article (CrossRef Link)

[2] X. Ge, S. Tu, G. Mao, C. X. Wang and T. Han, “5G ultra-dense cellular networks,” IEEE Wireless Communications, vol. 23, no. 1, pp. 72-79, March, 2016. Article (CrossRef Link)

[3] M. J. Yang, S. Y. Lim, H. J. Park and N. H. Park, "Solving the data overload: Device-to-device bearer control architecture for cellular data offloading,” IEEE Vehicular Technology Magazine, vol. 8, no. 1, pp. 31-39, March, 2013. Article (CrossRef Link)

[4] P. Phunchongharn, E. Hossain and D. Kim, "Resource allocation for device-to-device communications underlaying LTE-Advanced networks," IEEE Wireless Communications Magazine, vol. 20, no. 4, pp. 91-100, August, 2013. Article (CrossRef Link)

[5] F. Wang, C. Xu, L. Song and Z. Han, "Energy-efficient resource allocation for device-to-device underlay communication," IEEE Transactions on Wireless Communications, vol. 14, no. 4, pp. 2082-2092, April, 2015. Article (CrossRef Link)

[6] Y. Huang, A. Nasir, S. Durrani and X. Zhou, "Mode selection, resource allocation, and power control for D2D-enabled two-tier cellular network," IEEE Transactions on communications, vol. 64, no. 8, pp. 3534-3547, August 2016. Article (CrossRef Link)

[7] M. Jung, K. Hwang and S. Choi, "Joint mode selection and power allocation scheme for power-efficient device-to-device (D2D) communication," in Proc. of IEEE Conf. on Vehicular Technology Conference (VTC Spring). pp. 1-6, May 6-9, 2012. Article (CrossRef Link)

[8] H. Zhang, C. Jiang, X. Mao and H.H. Chen, "Distributed power control for D2D communications underlaying cellular network using Stackelberg game," in Proc. of IEEE Wireless Communications and Networking Conference (WCNC). pp. 1-5, March 11-27, 2017.

\section{Article (CrossRef Link)}

[9] G. Zhang, J. H, Wei, H. Li and G. Wang, "Device-to-device communications underlaying cellular networks," IEEE Transactions on Communications, vol. 61, no. 8, pp. 3541-3551, August, 2013. Article (CrossRef Link)

[10] L. Wang and H.Q. Wu, "Fast pairing of device-to-device link underlay for spectrum sharing with cellular users," IEEE Communications Letters, vol. 18, no. 10, pp. 1803-1806, October, 2014. Article (CrossRef Link)

[11] Q. Ye, M. Al-Shalash, C. Caramanis and J. G. Andrews, "Distributed resource allocation in device-to-device enhanced cellular networks," IEEE Transactions on Communications, vol. 63, no. 2, pp. 441-454, February, 2015. Article (CrossRef Link) 
[12] R. Yin, C. Zhong, G. Yu, Z. Zhang, K. K. Wong and X. Chen, “Joint spectrum and power allocation for D2D communications underlaying cellular networks," IEEE Transactions on Vehicular Technology, vol. 65, no. 4, pp. 2182-2195, April, 2016. Article (CrossRef Link)

[13] M. Jo, T. Maksymyuk, B. Strykhalyuk and C. H. Cho, "Device-to-device-based heterogeneous radio access network architecture for mobile cloud computing,” IEEE Wireless Communications, vol. 22, no. 3, pp. 50-58, July, 2015. Article (CrossRef Link)

[14] W. Wang, Y. Liu, Z. Luo, T. Jiang and Q. Zhang, “Toward cross-layer design for non-orthogonal multiple access: a quality-of-experience perspective,” IEEE Wireless Communications, January, 2018. Article (CrossRef Link)

[15] W. Wang, Y. Chen, Q. Zhang and T. Jiang, “A software-defined wireless networking enabled spectrum management architecture,” IEEE Communications Magazine, vol. 54, no. 1, pp. 33-39, January, 2016. Article (CrossRef Link)

[16] D. Zheng, C. He, L. Jiang, J. Ding and Q. Zhang, "Power optimization for D2D communication based on rate requirement in relay-assisted networks," In Proc of IEEE conf. on International Conference on Communication Workshop (ICCW), pp. 686-691, June 8-12, 2015. Article (CrossRef Link)

[17] Y. Han, A. Pandharipande and S. H Ting, "Cooperative decode-and-forward relaying for secondary spectrum access,” IEEE Transactions on Wireless Communications, vol. 8, no. 10, pp. 4945-4950, October, 2009. Article (CrossRef Link)

[18] B. Zhou, H. Hu, S. Huang and H-H. Chen, "Intracluster device-to-device relay algorithm with optimal resource utilization,” IEEE Transactions on Vehicular Technology, vol. 62, no. 5, pp. 2315-2326, June, 2013. Article (CrossRef Link)

[19] Y. Pei and Y.C. Liang, "Resource allocation for device-to-device communications overlaying two-way cellular networks,” IEEE Transactions on Wireless Communications, vol. 12, no. 7, pp. 3611-3621, July, 2013. Article (CrossRef Link)

[20] G. Zhang, K. Yang, S. Wu, X. Mei and Z. Zhao, "Efficient power control for half-duplex relay based D2D networks under sum power constraints," Wireless Network, vol. 21, no. 7, pp. 2345-2355, February, 2015. Article (CrossRef Link)

[21] T. Riihonen, S.Werner, and R.Wichman, "Hybrid full-duplex/half-duplex relaying with transmit power adaptation," IEEE Transactions on Wireless Communications, vol. 10, no. 9, pp. 3074-3085, September, 2011. Article (CrossRef Link)

[22] T. Kim and M. Dong, "An iterative Hungarian method to joint relay selection and resource allocation for D2D communications,” IEEE Wireless Communications Letters, vol. 3, no. 6, pp. 625-628, December, 2014. Article (CrossRef Link)

[23] H. Dun, F. Ye and S.H. Jiao, "Transmission power adaption for full-duplex relay-aided device-to-device communication,” Symmetry, vol. 9, no. 38, pp. 1-13, March, 2017.

Article (CrossRef Link)

[24] G. Zhang, K. Yang, P. Liu and J. Wei, "Power allocation for full-duplex relaying-based D2D communication underlaying cellular networks,” IEEE Transactions on Vehicular Technology, vol. 64, no. 10, pp.4911-4916, October, 2015. Article (CrossRef Link)

[25] Q. Li, M. Yu, A. P Pandharipande, X. Ge and J. Zhang, "Performance of virtual full-duplex relaying on cooperative multi-path relay channels," IEEE Transactions on Wireless Communications, vol. 15, no. 5, pp. 3628-3642, May, 2016. Article (CrossRef Link)

[26] Q. Li, M. Yu, A. Pandharipande and X. Ge, "Outage analysis of cooperative two-path relay channels,” IEEE Transactions on Wireless Communications, vol. 15, no. 5, pp. 3573-3578, May, 2016. Article (CrossRef Link)

[27] H. Dun, F. Ye, S.H. Jiao and D.D. Liu, "Power control for device-to-device communication with a hybrid relay mode in unequal transmission slots,” Electronics, vol. 7, no. 2, pp. 1-17, Jan, 2018. $\underline{\text { Article (CrossRef Link) }}$ 


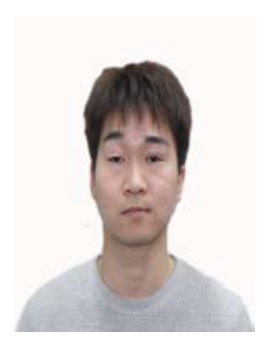

Hui Dun received his B.S. degree in communication engineering from Harbin Engineering University in 2014. He is currently working towards the Ph.D. degree in Harbin Engineering University. His research interests include LTE, SC-FDMA technology, D2D and wireless resource management.

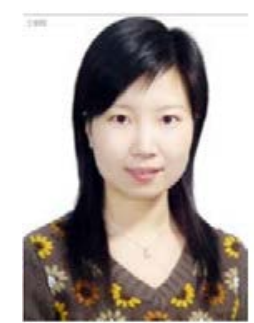

Fang Ye received the B.S. and Ph.D degrees in Electrical Information Engineering from Harbin Engineering University(HEU) in 2002 and 2006, respectively. She has been a teacher in Harbin Engineering University of China since 2002, and became an associate professor in 2007. During 2007-2008, she stayed in School of Electronics and Computer Science from University of Southampton as a visiting scholar. Now she is an IEEE member, a member of China Institute of Communications and a member of China Computer Federation. Her research interests include LTE technology, adaptive radio resource allocation technology and Cognitive radio.

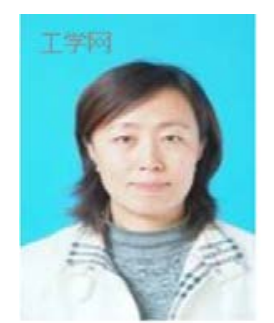

Shuhong Jiao received the B.S. and Ph.D degrees in Electrical Information Engineering from Harbin Engineering University(HEU) in 1991 and 2000, respectively. She has been a teacher in Harbin Engineering University of China since 1991, and became a professor in 2007. Now she serves as the national natural science foundation and the post-doctoral fund evaluation expert of the ministry of education. She has stayed the national institute of applied sciences in Lyon, France and Nanyang Technological University, Singapore as a visiting scholar. Her research interests include broadband signal detection, processing and identification, big data monitoring and analysis, image processing and analysis. 